\title{
Constraints for representing transforming entities in bio-ontologies
}

\author{
C. Maria Keet \\ KRDB Research Centre, Free University of Bozen-Bolzano, Italy \\ keet@inf.unibz.it
}

\begin{abstract}
Things change - develop, mature morph-but not everything in the same way. Representing this knowledge in ontologies faces issues on three fronts: what the category of the participating objects are, which type of relations they involve, and where constraints should be added. More precise distinctions can be made by using OntoClean's properties and a novel status property that is generalised from formal temporal conceptual data modeling. Criteria are identified, formulated in 17 additional constraints, and assessed on applicability for representing transformations more accurately. This enables developers of (bio-)ontologies to represent and relate entities more precisely, such as monocyte \& macrophage and healthy \& unhealthy organs.
\end{abstract}

\section{Introduction}

Much effort has been invested in development of ontologies, and in particular of bio-ontologies such as the Gene Ontology, Foundational Model of Anatomy, Cell Cycle, SNOMED and so forth $[1,2]$. This started from preliminary categorizations and vocabularies of biological entities that primarily focussed on endurants. With the maturing of (bio-)ontologies, ontology development tools, and ontology languages, this scope is broadening to temporal aspects. For instance, the Relation Ontology (RO) [3] contains the hitherto underused transformation_of and derives_from relations and several more evolution constraints have been identified for temporal conceptual modelling [4] that may find their way into domain ontologies. To be able to say one thing transforms into another, one has to identify the transforming entity $x$, the transformed entity $y$, and that the entity preserves its identity irrespective of the transformation while instantiating distinct classes at distinct points in time. What kind of entities are $x$ and $y$ instances of; phased sortals, roles, or merely different states? How should one deal with the temporality to achieve implementable knowledge bases that can handle representations of, and reasoning over, transforming entities? The aim of this paper is to characterise constraints on changing entities in more detail in a way so that it can aid a domain ontology developer to introduce or improve the representation of transforming entities, using an approach that is sufficiently generic to be extensible also to other types of change and to represent as much as possible about temporal aspects in the, to date, a-temporal commonly used ontology languages. We will commence with an analysis from an ontology development 
perspective to formulate a set of basic constraints (section 2). In section 3, we extract implicit ontological commitments from advances in temporal knowledge representation, which enables us to define additional constraints on the entities and the process of transformation. The resulting 17 constraints are assessed on their usefulness by reconsidering some recent problems in biomedical ontology development in section 4 . We discuss and conclude in section 5 .

\section{Analysis from an ontology development perspective}

\subsection{Preliminaries}

Transformations in the Relation Ontology (RO). Let us recollect the definition of the transformation_of relation in the RO [3]: "C transformation_of $C_{1}=$ [definition] $C$ and $C_{1}$ for all $c, t$, if $C c t$, then there is some $t_{1}$ such that $C_{1} c t_{1}$, and $t_{1}$ earlier $t$, and there is no $t_{2}$ such that $C c t_{2}$ and $C_{1} c t_{2}$. ." In an ontology engineering setting, this definition reveals two issues. First, it is ignorant of the distinction between the cases of unidirectional transformations versus where some instance of $C_{1}$ may, after transforming into $C$, transform back into $C_{1}$; e.g., the transformations of erythrocytes (red blood cells) into echinocytes and back again, and of a healthy organ into a non-healthy organ and back again. Second, and more important for developing logic-based ontologies (i.e., as artifact), the $\mathrm{RO}$ definition does not say how the entities undergoing transformation are able to change and yet keep their identity, other than through the use of the same variable ' $c$ '. This under-specification can lead to unintended models of the theory; e.g., a given particular remains unchanged, but either $C$ or $C_{1}$ changes due to increased understanding or correcting a representation error in the domain ontology. Clearly, this does not meet the intention of the authors of the above definition. To exclude such unintended models, we have to make explicit in the definition that the individual changes somehow. Let $a$ instantiate universals $C_{s}$ and $C_{t}$-with $s$ for source and $t$ for target the source is transformed into- at the two different times, then we have to assert that 'enough' properties are shared by $a \in C_{s}$ and $a \in C_{t}$ so that they can be identified as the same individual -in shorthand notation: $a_{s}={ }_{i} a_{t}$-while other properties $\pi_{1} \ldots \pi_{n}$ of $a$ are lost or gained so that after the transformation, $a$ instantiates a different universal. A basis for a generic definition might then be:

Definition 1 ( $C_{t}$ transformation_of $\left.C_{s}\right)$. Let $C_{t}$ be the target and $C_{s}$ the source universal, $x, y$ range over instances and $t_{0}, \ldots, t_{n}$ range over points in time, then $C_{t}(x)$ transformation_of $C_{s}(y)$ iff for all $x$, there exist $y, t_{0}, \ldots t_{n}$, if $C_{t}\left(x, t_{0}\right)$, then there is some $t_{1}$ such that $C_{s}\left(y, t_{1}\right), t_{1}<t_{0}, C_{s}$ and $C_{t}$ have the same identity criterion $\left(C_{s}={ }_{i} C_{t}\right), x$ and $y$ differ in at least one other property $\pi_{i}$, and there does not exist a $t_{2}$ such that $C_{t}\left(x, t_{2}\right)$ and $C_{s}\left(y, t_{2}\right)$.

In the next sections, we elaborate on this definition and elicit additional constraints to model and achieve the intended behaviour of transformation_of in ontologies and knowledge bases for as much as possible in the a-temporal setting of common ontology languages and tools for the Semantic Web. 
Basic notions from OntoClean. The main proposal in ontology to represent the entities involved in transformations is that of phased sortals. Postulating that $C_{t}$ and $C_{s}$ are phased sortals (or another category), brings forward constraints on the participating entities of the transformation relation that, in turn, may affect constraints on the relation itself, and certainly influences ontology development. To arrive at the point where we can specify unambiguously its how and where, we first summarise a section of $[5,6]$ that form the basis of the OntoClean methodology ${ }^{1}[9]$. The relevant aspects for the current scope are the notion of identity, the meta-property rigidity, and identity criteria. Identity of an instance, and by extension also the universal it instantiates, focuses on the problems of distinguishing an instance of one class from other instances of that class by means of a characteristic property, which is unique for that whole instance, and is a relation that each thing has to itself and to nothing else; consequently, the property is essential. The property rigidity is based on this notion of essential property; two of the four modes in [5] are relevant for the current scope:

Definition $2(+\mathbf{R})$. A rigid property $\phi$ is a property that is essential to all its instances, i.e., $\forall x \phi(x) \rightarrow \square \phi(x)$

Definition $3(\sim \mathbf{R})$. An anti-rigid property $\phi$ is a property that is not essential to all its instances, i.e., $\forall x \phi(x) \rightarrow \neg \square \phi(x)$

For instance, the properties being a Patient and being a Caterpillar are $\sim \mathrm{R}$ and being a Person and being a Herbivore are $+\mathrm{R}$ that may subsume Patient and Caterpillar, respectively. Objects can keep their identity through the changes it may undergo during its lifetime, thereby exhibiting different properties at different times; thus, we consider diachronic identity (cf. synchronic identity) which is about establishing that two entities are the same at two different points in time. Setting aside how we can identify instances - it is philosophically difficult [10], but one can always follow common practice and use either identifiers or a collection of OWL object and data properties - the instances and its corresponding universal have identity criteria (IC), which are both necessary and sufficient for identity $[5,6]$; properties carrying an IC are called sortals and only $+\mathrm{R}$ properties are sortal properties. In addition, a rigid property $\phi$ either carries the necessary IC $\Gamma$ or it carries the sufficient IC $\Gamma$ (see also [5] definitions 5 and 6 ). Now we can introduce two other definitions from [5], which are important for phased sortals.

Definition $4(+\mathbf{I})$. A property that is not rigid carries an IC $\Gamma$ iff it is subsumed by a rigid property carrying $\Gamma$.

Definition $5(+\mathbf{O})$. A property $\phi$ supplies an IC $\Gamma$ iff $i)$ it is rigid; ii) it carries $\Gamma$; and iii) $\Gamma$ is not carried by all the properties subsuming $\phi$.

\footnotetext{
${ }^{1}$ There are some refinements but they do not affect the principal method of categorisation (in Fig.1, below). Moreover, the extension toward OntoClean 2.0 [7] with temporal aspects fit well with the temporal language we build upon in section 3 (as has been demonstrated by [8] for rigidity and part-whole relations).
} 
Thus, an $+\mathrm{O}$ property brings in its own identity criterion as opposed to just carrying it; conversely, properties that do not carry identity or do not supply identity are marked with -I and -O, respectively. Fig.1 summarises a classification of property kinds based on the R, O, and I meta-properties.

\begin{tabular}{|c|c|c|c|c|c|}
\hline$+\mathrm{O}$ & +1 & $+\mathrm{R}$ & $+D$ & Type & \multirow{6}{*}{ Sortal } \\
\hline \multirow[b]{2}{*}{-0} & \multirow[b]{2}{*}{+1} & \multirow[b]{2}{*}{$+R$} & $\frac{-D}{+D}$ & \multirow[b]{2}{*}{ Quasi-Type } & \\
\hline & & & $\frac{-D}{-D}$ & & \\
\hline$-\mathrm{O}$ & +1 & $\sim R$ & $+D$ & Material role & \\
\hline$-\mathrm{O}$ & +1 & $\sim R$ & $-D$ & Phased sortal & \\
\hline$-\mathrm{O}$ & +1 & $\neg \mathrm{R}$ & $+D$ & Mixin & \\
\hline
\end{tabular}

Fig. 1. Ontological properties [5] (non-sortals omitted). $+\mathrm{R}$ : rigid; $\sim \mathrm{R}$ anti-rigid and $\neg \mathrm{R}$ semi-rigid, $+\mathrm{O}$ own identity supplied, $+\mathrm{I}$ identity carried, and $+\mathrm{D}$ dependent.

\subsection{Characterising the transforming entities}

Given the classification (Fig.1), this translates into the following set of basic constraints CT1-CT5 for phased sortals. CT2 ensures that the entity that changes local IC when going from one 'phase' to another, still can be identified as the same entity (thanks to property inheritance from $C_{p}$ ). CT3 can be derived from CT1, CT2, and Definition 4.

(CT1) A phased sortal does not supply an IC, i.e., -O

(CT2) A phased sortal must be subsumed by $C_{p}$ that has $+\mathrm{O}$

(CT3) A phased sortal carries an IC, i.e., +I

(CT4) A phased sortal is a sortal

(CT5) A phased sortal is anti-rigid, i.e., $\sim \mathrm{R}$

It is now straightforward to demonstrate that if $C_{t}$ and $C_{s}$ of the transformation_of relation are both categorised as phased sortals, then:

(CT6) $C_{t}$ and $C_{s}$ both must be subsumed by $C_{p}$

As we shall see later with the examples, CT6 is particularly important. It immediately follows from Definition 5, the classification (Fig.1), and CT6 that $C_{p}$ must be a type (CT7). Further, phased sortals together with the transformation_of relation cover the implicit requirement that phased sortals never can occur 'alone', because in order to phase, one needs at least 2 phased sortals (CT8).

(CT7) $C_{p}$ must be a type $(+\mathrm{O}+\mathrm{I}+\mathrm{R})$

(CT8) Each type that subsumes phased sortals, which are related through the transformation_of relation, must subsume at least two phased sortals

It is possible that $C_{t}$ and $C_{s}$ represent universals for different 'states' of a common subsumer $C_{p}$, provided the states are distinct enough. However, how does state differ from phase? Intuitively, phases succeed one another and have no 
circularity, whereas states (e.g., sensu DOLCE [11]) permit going back- and forward between alternating states. Let us take the latter assumption, then if $C_{t}$ transformation_of $C_{s}$ and $C_{t}$ and $C_{s}$ are categorised as states, then the following constraints must hold:

(CT9) $C_{t}$ and $C_{s}$ must carry identity $(+\mathrm{I})$

(CT10) If $C_{t}$ is a transformation of $C_{s}$, then it is possible, but not necessary, that at a later point in time $C_{t}$ transforms back into $C_{s}$

(CT11) $C_{t}$ and $C_{s}$ have meta-properties that are either $\sim \mathrm{R}$ or $+\mathrm{R}$

This reduces $C_{t}$ and $C_{s}$ to being either types, quasi-types, material roles, or phased sortals. They cannot be roles, because an instance can have more than one role at the same time (e.g., Patient and Employee), thereby violating Definition 1. $C_{s}$ and $C_{t}$ cannot be types either, because then one cannot ensure a common IC for diachronic identity. They can be both quasi-types $(-\mathrm{O}+\mathrm{I}+\mathrm{R})$, which is a kind that enables one to group entities based on properties that do not affect identity of $C_{s}$ and $C_{t}$ (because of -O), such as being a Herbivore. We add CT12 in order to prevent $C_{t}$ and $C_{s}$ to be also types or roles and we make explicit an assumption that transformation of phased sortals is unidirectional (CT13).

(CT12) If $C_{t}$ and $C_{s}$ are categorised as states, they are neither both types nor both roles

(CT13) If $C_{t}$ is a transformation of $C_{s}, C_{t}$ and $C_{s}$ are phased sortals, then it is not possible that at a later point in time $C_{t}$ is a transformation of $C_{s}$, i.e., $C_{t}$ does not transform back

Thus, based on foundational notions of Ontology, CT1-CT13 offers a more precise catergorisation for the relata of the transformation_of, as well as their position in a taxonomy.

\section{Constraints from temporal conceptual modelling}

The challenge of how to represent changing entities has been investigated also from formal and engineering perspectives, most notably with formal temporal conceptual data modelling. Artale et al [4] have formalised the well-known core elements of temporal databases in $\mathcal{D} \mathcal{L R}_{\mathcal{U S}}$, a temporal description logic that is an expressive fragment of the first order temporal logic $L^{\{\text {Since, Until }\}}$, and a corresponding $\mathcal{E R}_{V T}$ temporal conceptual data modelling language that extends EER. However, because it is intended for conceptual data modelling, they do not take into account the kind of classes like phased sortal-hence, nor the possible consequences on the logical implications either-but they include evolution constraints other than transformation, such as dynamic extension and generation. Therefore, we only consider their notion of status classes here. Status is associated to a class to log the evolving status of membership of each object in the class and the relation between the statuses. The four possible statuses are scheduled, active (for backwards compatibility with an a-temporal class), suspended, and disabled (based on [12]), with various formalised constraints; e.g., 
that an object that is member of the disabled class can never become member of its active class again, existence - where exists subsumes scheduled, active, and suspended-persist until disabled, and exists is disjoint from disabled [4]. In this setting, one can assert, e.g., that Caterpillar is temporally related to Butterfly: when at $t_{0}$ object $o \in$ Caterpillar (and $o \in$ Scheduled-Butterfly) starts transforming into an instance of Butterfly, then we have at the next time $(\oplus$ in $\left.\mathcal{D} \mathcal{L}_{\mathcal{U S}}\right)$ transformation at $t_{1}\left(\right.$ with $\left.t_{0}<t_{1}\right)$ that $o \in$ Disabled-Caterpillar and $o \in$ Butterfly. Thus, status allows us to add additional constraints to $C_{t}$ and $C_{s}$.

Status property $\mathbf{S}$ and additional constraints. Given the formal semantics of status classes [4], we introduce the property $\mathrm{S}$ and emphasize the core notions of the four status classes and what holds for their respective instances:

Definition $6(+\mathbf{S})$. A property $\phi$ has status active at time $t$ iff $\phi(x)$ holds at time $t$.

Definition 7 (-S). If a property $\phi$ has status scheduled at time $t$ then $\phi(x)$ holds at some time $t_{0}$, for $t_{0}>t$.

Definition $8(\sim \mathbf{S})$. If a property $\phi$ has status suspended at time then $\phi(x)$ holds at some time $t_{0}$, with $t_{0}<t$.

Definition $9(\neg \mathbf{S})$. A property $\phi$ has status disabled at time $t$ iff $\phi$ holds at some time $t_{0}$, with $t_{0}<t$, and for all $t^{\prime}$, such that $t^{\prime} \geq t, \phi(x)$ does not hold.

This enables us to specify the following constraints for the transformation_of relation and its relata, when the instance cannot transform back:

(CT14) $C_{s}$ has $+\mathrm{S}$ at the time of transformation and $\neg \mathrm{S}$ after transformation (CT15) $C_{t}$ has $-\mathrm{S}$ at the time of transformation and $+\mathrm{S}$ after transformation

If the entity can transform back, then CT14 and CT15 have to be replaced with:

$\left(\mathrm{CT} 14^{\prime}\right) C_{s}$ has $+\mathrm{S}$ at the time of transformation and either $\neg \mathrm{S}$ or $\sim \mathrm{S}$ after transformation

$\left(\mathrm{CT} 15^{\prime}\right) C_{t}$ has either $-\mathrm{S}$ or $\sim \mathrm{S}$ at the time of transformation and $+\mathrm{S}$ after transformation

Thus, theoretical contributions in formal conceptual modelling for temporal databases have the ontological commitment for CT14' and CT15', whereas transformation_of was ignorant about this aspect. One could argue S is ontologically awkward for it hints toward intentionality, but representing transformations does consider that anyway, and therefore it is preferable to make this explicit. In addition, bio-ontologies are, or should be, represented in some formal ontology language; using $\mathrm{S}$ then provides a useful mechanism to represent precisely and explicitly such implicit assumptions about the subject domain, thereby pushing for closer analysis by ontology developers as well as ontological investigation into intentional aspects in a temporal setting. In addition, it offers a 
way to deal with some underlying ideas about temporal aspects yet representing it in a commonly used a-temporal ontology language such as OWL.

CT14 and CT15 versus CT14' and CT15' leads to two distinct sets of constraints on the position of classes in a taxonomy. Let $\forall x(\phi(x) \rightarrow \psi(x))$, henceforth abbreviated as $\phi \rightarrow \psi$, a class' property $\mathrm{S}$ indicated with superscript $\phi^{+}$, $\phi \sim$ and so forth, and 'in the past' denoted with a " $\diamond$ ", then we can adjust Artale et al's constrains for CT14' \& CT15' as shown in (1-5). When transformation back is not permitted, then (1), (4), (5), and (6) hold; that is, suspended is not permitted, such that $(2)$ is not applicable and $\psi^{\sim}$ is removed from $(3)$ and therefore replaced by $(6)$.

$$
\begin{aligned}
& \phi^{+} \rightarrow \psi^{+} \\
& \phi^{\sim} \rightarrow \psi^{\sim} \vee \psi^{+} \\
& \phi\urcorner \rightarrow \psi\urcorner \vee \psi^{\sim} \vee \psi^{+} \\
& \phi^{-} \rightarrow \neg \psi^{\urcorner} \\
& \left.\psi\urcorner \wedge \diamond \phi^{+} \rightarrow \phi\right\urcorner \\
& \phi\urcorner \rightarrow \psi\urcorner \vee \psi^{+}
\end{aligned}
$$

Combining this with the meta-properties of phased sortals, then the subsumption constraints (1) \& (4), CT6, CT14 \& CT15 imply $C_{p}^{+}$, because always one of the phased sortals subsumed by $C_{p}$ is active. Regarding permitting suspension, $\sim \mathrm{S}$, then $C_{p}^{+}$is also implied, because of (1), (2), (4), CT14' \& CT15'.

\section{Typical biomedical examples re-examined}

In this section we assess if and how the proposed constraints suffice for modelling transformations in biology and biomedicine, using monocytes/macrophages and pathological transformations as examples.

Monocyte/macrophage: alternating states or phased sortals? Multiple cell types in the human body, most notably those involved in hematopoiesis, can be pluri-potent stem cells, progenitor cells that differentiate, mature, and change in other ways, i.e., they seem to transform and are candidates for being categorised as phased sortals. However, looking at the details of the cells and processes, they are neither necessarily transformations nor necessarily phased sortals. For instance, progenitor cells undergo cell division and differentiation up to their final types, which is a non-deterministic process up to the point that the cells cease to be progenitor cells. But what about those final types? Here, we analyse the deterministic transformation from monocyte to macrophage, which, curiously, is also called in one compound term "monocyte/macrophage" or "monocyte-macrophage" in recent scientific literature (e.g. [13]) as if we have a cellular version of the Morning/Evening Star. Summarizing the scientific knowledge, Monocyte is part of Blood [2], the cells dock into tissues to transform into macrophages, and are considered part of the ImmuneSystem [14]. In the FMA taxonomy, Monocyte is subsumed by NongranularLeukocyte that is subsumed by Leukocyte, whereas Macrophage is directly subsumed by Leukocyte [2]. Monocytes are considered to be the end stage of the differentiation, and they can change into different types of macrophages, such as microglia (macrophages 
located in the brain), so that CT14 \& CT15 hold. A particular monocyte transforms into a macrophage only when it leaves the blood stream into tissue, changing from "APC, circulating" to "APC, tissue resident", respectively [15] (APC $=$ Antigen Presenting Cell). A phenotypic difference is that macrophages also stimulates $\mathrm{T}$ helper cells [15] whereas both perform phagocytose of foreign organisms. The processes of change involves, among others, physiological response (chemical \& DNA) on mechanical pressure [13] and response on the molecule platelet-derived $\alpha$-chemokine PF4 [16]. Three possible modelling options have been proposed to represent the transformation of monocyte to macrophage, which we reconsider here. Option 1: Monocyte and macrophage are phased sortals. The problem with this approach is that there is no known suitable $C_{p}$ that subsumes both of them only and directly (Leukocyte subsumes a range of other cells), i.e., violating CT6, CT7, CT8. There are multiple similar cases with hemal cells, which, if decided upon nevertheless, require additions of many more concepts that do not have a (known) corresponding universal in reality. Option 2: Monocyte and macrophage are different states that instances of $C_{p}$ can have, because of the alternative functional states in the adult stage where each one is best adapted to its own environment: one residing in a fluid with the optimal shape of a sphere, the other in tissue with docking extensions on the cell surface, whilst remaining the same individual cell (cf. multi-cellular life cycles) instantiating one universal throughout the changes. Classifying the two states as distinct classes based on these differences is pursued by the Physiome project [15]. Both are APCs and phagocytes, which are candidates for the $=_{i}$ in Definition 1. Ascertaining $\pi_{1} \ldots \pi_{n}$, they could be location and shape (attributions $-\mathrm{I} \sim \mathrm{R}$ ) or the property of stimulation of $\mathrm{T}$ helper cells for macrophages, or their differences in activated genes. This approach satisfies CT1, CT3, CT5, CT9, CT10, CT11, CT12, CT14 \& CT15. Option 3: Take Monocyte and Macrophage as universals, place them somewhere in the taxonomy of cell types and create a new relationship type $R$ to relate them. This does not guarantee diachronic identity of the instances and is therefore inappropriate. Clearly, with the current available information about the mechanisms of transformation from monocyte to macrophage, option 2 offers the more accurate representation.

Pathological transformations. Smith et al [3] include pathological transformations, which complicates both the permissible relata and the transformation _ of relation itself for two reasons. First, it is ambiguous if the pathological entity may transform back to its healthy form and if this should be under the assumption that nature takes its course or if it also permits medical intervention. Second, it is not the case that for all instances that are transformed into a pathological entity they either all can or all cannot transform back; e.g., 5 out of 100 lung cancer patients survive and keep their lungs. It is true that for all carcinomatous lungs there must have been healthy lungs, but the inverse does not hold for all instances. Moreover, the relata cannot be phased sortals because there is no common subsumer $C_{p}$ for the healthy and carcinomatous lungs in extant ontologies; e.g., the FMA commits to the "canonical" case, i.e., the assumption 
is that Lung refers to healthy lungs already. Given these considerations, they lead to the combination of constraints where CT9-CT12 hold together with either CT14 \& CT15 for healthy \& pathological entities of non-curable diseases or $\mathrm{CT} 14^{\prime} \& \mathrm{CT} 15^{\prime}$ for curable diseases.

\section{Discussion and conclusions}

Regarding the transformation_of relation, the basic questions to answer for representing the universals in the subject domain in a domain ontology are: what transforms, how does it transform, and why does it transform? We focussed on the first two questions. Definition 1 in conjunction with constraints CT1-CT15' facilitate the analysis of transforming entities as well as the transformations themselves. For requirements on the participating endurants, the constraints for phased sortals to be subsumed by a type may be too restrictive for representing transforming biological universals most accurately for the main reasons that often either no such $C_{p}$ is known to exist in reality that readily fits this requirement or it does exist but the criterion that supplies identity is difficult to establish; put differently, states and quasi-types seem to be more adequate for the participating continuants. Nevertheless, phased sortals remain useful kinds for representing stages in organism's life cycle as well as transformations of progenitor cells to differentiated cells.

The property status S helps in understanding and representing more precisely how the instances transform, thanks to the constraints it imposes both on status change during transformation and the universals' position in the taxonomy. For the biomedical domain, this is particularly useful for transformations involving pathological entities. More generally, several combinations and exclusions of constraints can be given:

i. Phased sortals, unidirectional transformation: CT1-CT8, CT13, CT14, CT15;

ii. States (including quasi-types), unidirectional transformation: CT1-CT9, CT11-CT15;

iii. States (including quasi-types), transformation back is possible: CT1-CT13, CT14', CT15';

iv. Pathological transformations, terminal disease: see constraints point ii, permit status change from -S directly into $\neg \mathrm{S}$;

v. Pathological transformations, reversal possible: see constraints point iii, permit status change from -S directly into $\neg \mathrm{S}$.

One can make a further distinction for point $\mathrm{v}$ and define two sub-types of the transformation relation, which is to distinguish between self-healing transformations and those that require medical intervention, i.e., distinguishing between natural transformations and human-mediated transformations.

To actually use the different constraints and options for representing the transformation_of relation, it will be helpful to make the distinctions explicit also in the ontology development software by bringing representation choices of the properties to the foreground, which could be added to OntoClean.

Concluding, to represent changing entities more precisely, we took into ac- 
count the kind of the participating entities and proposed that more precise distinctions can be made based on some core ideas of the OntoClean approach together with the notion of a status property that was generalised from temporal conceptual data modeling. This resulted in 17 additional constraints, which were assessed on applicability to bio-ontologies by analysing typical examples such as monocyte \& macrophage. Currently, we are investigating implications of the interactions between OntoClean's property kinds, the status property, and temporal constraints in $\mathcal{D} \mathcal{L} \mathcal{R}_{\mathcal{U}}$, and in future works we may consider more complex time modeling, such as with GFO [17].

Acknowledgments. The author would like to thank Alessandro Artale and Barry Smith for useful comments on an earlier draft.

\section{References}

1. Gene Ontology Consortium, .: The Gene Ontology GO database and informatics resource. Nucleic Acids Research 32(1) (2004) D258-D261

2. Rosse, C., Mejino Jr, J.L.V.: A reference ontology for biomedical informatics: the foundational model of anatomy. J. of Biomedical Informatics 36(6) (2003) 478-500

3. Smith, B., et al.: Relations in biomedical ontologies. Genome Biol. 6 (2005) R46

4. Artale, A., Parent, C., Spaccapietra, S.: Evolving objects in temporal information systems. Annals of Mathematics and Artificial Intelligence 50(1-2) (2007) 5-38

5. Guarino, N., Welty, C.: A formal ontology of properties. In: Proc. of EKAW '00. LNCS, Springer (2000)

6. Guarino, N., Welty, C.: Identity, unity, and individuality: towards a formal toolkit for ontological analysis. In: Proc. of ECAI-2000, IOS Press, Amsterdam (2000)

7. Welty, C., Andersen, W.: Towards ontoclean 2.0: a framework for rigidity. Journal of Applied Ontology 1(1) (2005) 107-11

8. Artale, A., Guarino, N., Keet, C.M.: Formalising temporal constraints on partwhole relations. In: Proc. of KR'08, AAAI Press (2008) 673-683

9. Guarino, N., Welty, C.: An overview of OntoClean. In Staab, S., Studer, R., eds.: Handbook on ontologies. Springer Verlag (2004) 151-159

10. Noonan, H.: Identity. In Zalta, E.N., ed.: The Stanford Encyclopedia of Philosophy. Fall 2008 edn. (2008) http://plato.stanford.edu/archives/fall2008/entries/identity/.

11. Masolo, C., Borgo, S., Gangemi, A., Guarino, N., Oltramari, A.: Ontology library. WonderWeb Deliverable D18 (ver. 1.0, 31-12-2003). (2003)

12. Spaccapietra, S., Parent, C., Zimanyi, E.: Modeling time from a conceptual perspective. In: Proceedings of CIKM98. (1998)

13. Yang, J.H., Sakamoto, H., Xu, E.C., Lee, R.: Biomechanical regulation of human monocyte/macrophage molecular function. Am. J. Path. 156 (2000) 1797-1804

14. Hoffbrand, A.V., Pettit, J.E.: Atlas of Clinical Heamatology. 3 edn. Elsevier (2000)

15. Hunter, P., Borg, T.: Integration from proteins to organs: The physiome project. Nature 4(3) (2003) 237-243

16. Scheuerer, B., et al.: The cxc-chemokine platelet factor 4 promotes monocyte survival and induces monocyte differentiation into macrophages. Blood 95(4) (2000) $1158-1166$

17. Herre, H., Heller, B.: Ontology of time and situoids in medical conceptual modeling. In: Proc. of AIME 2005. Volume 3581 of LNAI., Springer-Verlag (2005) 266-275 